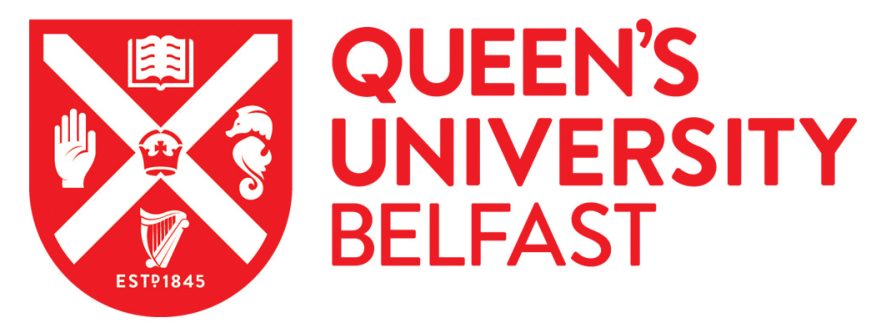

\title{
Ultra-bright Optical Transients are Linked with Type Ic Supernovae
}

Pastorello, A., Smartt, S. J., Botticella, M. T., Maguire, K., Fraser, M., Smith, K., Kotak, R., Magill, L., Valenti, S., Young, D. R., Gezari, S., Bresolin, F., Kudritzki, R., Howell, D. A., Rest, A., Metcalfe, N., Mattila, S., Kankare, E., Huang, K. Y., ... Wynn-Williams, C. G. (2010). Ultra-bright Optical Transients are Linked with Type Ic

Supernovae. The Astrophysical Journal Letters, 724(1), L16- L21. https://doi.org/10.1088/2041-8205/724/1/L16

Published in:

The Astrophysical Journal Letters

Document Version:

Publisher's PDF, also known as Version of record

Queen's University Belfast - Research Portal:

Link to publication record in Queen's University Belfast Research Portal

Publisher rights

Copyright 2010. The American Astronomical Society. All rights reserved. Printed in the U.S.A.

\section{General rights}

Copyright for the publications made accessible via the Queen's University Belfast Research Portal is retained by the author(s) and / or other copyright owners and it is a condition of accessing these publications that users recognise and abide by the legal requirements associated with these rights.

Take down policy

The Research Portal is Queen's institutional repository that provides access to Queen's research output. Every effort has been made to ensure that content in the Research Portal does not infringe any person's rights, or applicable UK laws. If you discover content in the Research Portal that you believe breaches copyright or violates any law, please contact openaccess@qub.ac.uk. 


\title{
ULTRA-BRIGHT OPTICAL TRANSIENTS ARE LINKED WITH TYPE Ic SUPERNOVAE
}

\author{
A. Pastorello ${ }^{1}$, S. J. Smartt ${ }^{1}$, M. T. Botticella ${ }^{1}$, K. Maguire $^{1}$, M. Fraser ${ }^{1}$, K. Smith ${ }^{1}$, R. Kotak ${ }^{1}$, L. Magill ${ }^{1}$,

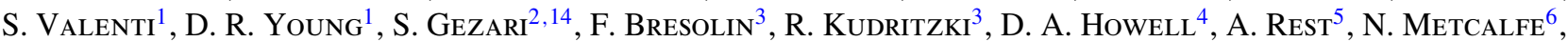

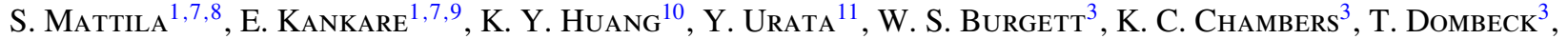 \\ H. Flewelling ${ }^{3}$, T. Grav ${ }^{2}$, J. N. Heasley ${ }^{3}$, K. W. HodapP ${ }^{3}$, N. Kaiser ${ }^{3}$, G. A. Luppino ${ }^{3}$, R. H. Lupton ${ }^{12}$, E. A. Magnier ${ }^{3}$,

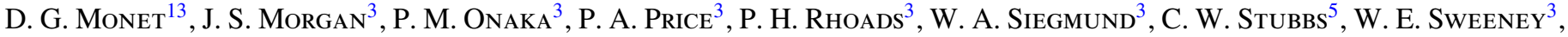 \\ J. L. TONRY ${ }^{3}$, R. J. WAINSCOAT ${ }^{3}$, M. F. WATERSON ${ }^{3}$, C. WATERS ${ }^{3}$, AND C. G. WyNN-WILLIAMS ${ }^{3}$ \\ ${ }^{1}$ Astrophysics Research Centre, School of Mathematics and Physics, Queen's University Belfast, Belfast BT7 1NN, UK; a.pastorello@qub.ac.uk \\ ${ }^{2}$ Department of Physics and Astronomy, Johns Hopkins University, 3400 North Charles Street, Baltimore, MD 21218, USA \\ ${ }^{3}$ Institute for Astronomy, University of Hawaii at Manoa, Honolulu, HI 96822, USA \\ ${ }^{4}$ Las Cumbres Observatory Global Telescope Network and the Department of Physics, University of California, Santa Barbara, CA 93117, USA \\ ${ }^{5}$ Department of Physics, Harvard University, Cambridge, MA 02138, USA \\ ${ }^{6}$ Department of Physics, University of Durham, South Road, Durham DH1 3LE, UK \\ ${ }^{7}$ Tuorla Observatory, Department of Physics and Astronomy, University of Turku, Piikkiö, FI 21500, Finland \\ ${ }^{8}$ Stockholm Observatory, Department of Astronomy, AlbaNova University Center, SE 10691 Stockholm, Sweden \\ ${ }^{9}$ Nordic Optical Telescope, Apartado 474, E-38700 Santa Cruz de La Palma, Spain \\ ${ }^{10}$ Academia Sinica Institute of Astronomy and Astrophysics, Taipei 106, Taiwan \\ ${ }^{11}$ Institute of Astronomy, National Central University, Chung-Li 32054, Taiwan \\ ${ }^{12}$ Department of Astrophysical Sciences, Princeton University, Princeton, NJ 08544, USA \\ ${ }^{13}$ US Naval Observatory, Flagstaff Station, Flagstaff, AZ 86001, USA \\ Received 2010 August 16; accepted 2010 September 9; published 2010 October 25
}

\begin{abstract}
Recent searches by unbiased, wide-field surveys have uncovered a group of extremely luminous optical transients. The initial discoveries of SN 2005ap by the Texas Supernova Search and SCP-06F6 in a deep Hubble pencil beam survey were followed by the Palomar Transient Factory confirmation of host redshifts for other similar transients. The transients share the common properties of high optical luminosities (peak magnitudes $\sim-21$ to -23 ), blue colors, and a lack of $\mathrm{H}$ or $\mathrm{He}$ spectral features. The physical mechanism that produces the luminosity is uncertain, with suggestions ranging from jet-driven explosion to pulsational pair instability. Here, we report the most detailed photometric and spectral coverage of an ultra-bright transient (SN 2010gx) detected in the Pan-STARRS 1 sky survey. In common with other transients in this family, early-time spectra show a blue continuum and prominent broad absorption lines of O II. However, about 25 days after discovery, the spectra developed type Ic supernova features, showing the characteristic broad Fe II and Si II absorption lines. Detailed, post-maximum follow-up may show that all SN 2005ap and SCP-06F6 type transients are linked to supernovae Ic. This poses problems in understanding the physics of the explosions: there is no indication from late-time photometry that the luminosity is powered by ${ }^{56} \mathrm{Ni}$, the broad light curves suggest very large ejected masses, and the slow spectral evolution is quite different from typical Ic timescales. The nature of the progenitor stars and the origin of the luminosity are intriguing and open questions.
\end{abstract}

Key words: supernovae: general - supernovae: individual (SN 2010gx, SCP-06F6, SN 2005ap)

Online-only material: color figures

\section{INTRODUCTION}

The discovery of unusual optical transients is a goal of modern surveys. Focused supernova searches (e.g., the Texas Supernova Search) or all-sky surveys, such as the Panoramic Survey Telescope \& Rapid Response System (Pan-STARRS), the Catalina Real-time Transient Survey (CRTS), the Palomar Transient Factory (PTF), and Skymapper are expected to discover a large number of new types of stellar explosions in the next years. The preliminary results are remarkable, and newly discovered transients are revolutionizing our knowledge of stellar explosions. Ultra-bright supernovae ( $\mathrm{SNe}$ ) associated with faint and, presumably, metal-poor host galaxies are the most spectacular recent discoveries (Quimby et al. 2007; Gezari et al. 2009; Miller et al. 2009; Gal-Yam et al. 2009; Young et al. 2010).

The field has moved quickly, prompted by the unusual transient SCP-06F6, discovered in the Hubble Space Telescope

\footnotetext{
${ }^{14}$ Hubble Fellow.
}

Cluster Supernova Survey (Barbary et al. 2009). Its light curve was symmetric, with a $\sim 100$ day rise time in the observed frame. The spectrum showed broad absorption features and the transient was associated with no obvious host galaxy (although a weak source, $1^{\prime \prime} .5$ from the transient, was marginally detected at magnitude $z \sim 25.8$ ). ${ }^{15}$ Without robust constraints on the absolute magnitude for this transient, even the discrimination between Galactic and extra-galactic origin was uncertain. Possible scenarios proposed by Barbary et al. (2009) for SCP-06F6 were an outburst of a Galactic C-rich white dwarf (WD), a broad absorption lines quasar or a micro-lensing event, but none of them was fully convincing. Assuming that the broad features in the spectra of SCP-06F6 were the $\mathrm{C}_{2}$ Swan bands, Gänsicke et al. (2009) tentatively fixed the redshift at $z=0.14$, implying an absolute peak magnitude of about -18 , suggesting an SN-like explosion of a C-rich Wolf-Rayet (WR) star. A tidal disruption of a C-rich star by a black hole (Rosswog et al. 2009; Soker

${ }^{15}$ Unless specified, magnitudes are in the $\mathrm{AB}$ system. 
et al. 2009), a Galactic WD-asteroid merger, and a type Ia SN in a dense, C-rich wind produced by a companion star (Soker et al. 2009) were also proposed as alternative explanations.

A few events have recently been discovered sharing observed properties with SCP-06F6. Data for a total sample of six objects have been presented by Quimby et al. (2010b). One of them was SN 2005ap, an enigmatic object originally presented in Quimby et al. (2007) and classified as a peculiar, overluminous SN IIL. Through the detection of narrow interstellar Mg II lines, Quimby et al. (2010b) have definitely proved that these transients are not located in the Galaxy or in the Local Group, but are relatively distant objects, with redshifts between 0.26 and 1.19. Consequently, they are extremely luminous, with $u$-band absolute magnitudes spanning between -22 and -23 . On the basis of the lack of any evidence of a slope consistent with ${ }^{56} \mathrm{Co}$ decay in the late-time light curve of both SCP-06F6 and SN 2005ap, Quimby et al. (2010b) favored either a pulsational pair-instability outburst scenario, or core-collapse SNe powered by rapidly rotating young magnetars.

Unfortunately, follow-up observations collected so far for these transients and the information available for properly studying and modeling their data have been incomplete. The discovery of a relatively nearby object of this class caught early and followed in detail, has provided us with a new opportunity to study the energy output and spectral evolution of one of nature's brightest explosions.

\section{THE DISCOVERY OF SN 2010GX}

The CRTS team (Drake et al. 2009) first announced the discovery of an optical transient (CSS100313:112547-084941) at R.A. $=11: 25: 46.71$ and decl. $=-08: 49: 41.4$, on images obtained on 2010 March 13 (magnitude 18.5; Mahabal et al. 2010). Its optical spectrum showed a blue, featureless continuum, and the initial redshift determination $(z=0.17)$ was later corrected by the same authors to $z=0.23$ (Mahabal \& Drake 2010).

On the following day, Quimby et al. (2010a) reported the independent discovery by the PTF survey (Rau et al. 2009; Law et al. 2009) of the same variable source (labeled as PTF10cwr) at several epochs between March 5 and 16, while no object was seen on March 4.27 UT to a limiting magnitude of 20.4. Optical spectra on March 18.27 UT showed that PTF10cwr was a luminous SN similar to the ultra-bright SN 2005ap (Quimby et al. 2007). The spectrum showed broad features attributed to O II (Quimby et al. 2010a). The presence of narrow lines attributed to a host galaxy allowed them to estimate the redshift to $z=0.23$.

In the course of the Pan-STARRS 1 Telescope (PS1) $3 \pi$ survey, we recovered the transient (PS1-1000037, hereafter SN 2010gx) between March 12 and 17 showing that its luminosity was still rising (Pastorello et al. 2010). Pastorello et al. (2010) noted the presence of a faint host galaxy in archive Sloan Digital Sky Survey (SDSS) images (SDSS J112546.72-084942.0) with magnitudes $g=22.7, g-r=0.3$ and confirmed the Quimby et al. (2010a) redshift estimate of $z=0.23$. At this redshift, the $g$-band absolute magnitude of the host galaxy is about -18 , similar to that of the LMC.

\section{OBSERVATIONS}

\subsection{Photometry}

We carried out an extensive ugriz photometric follow-up campaign of SN 2010gx using the telescopes listed in Table 1.

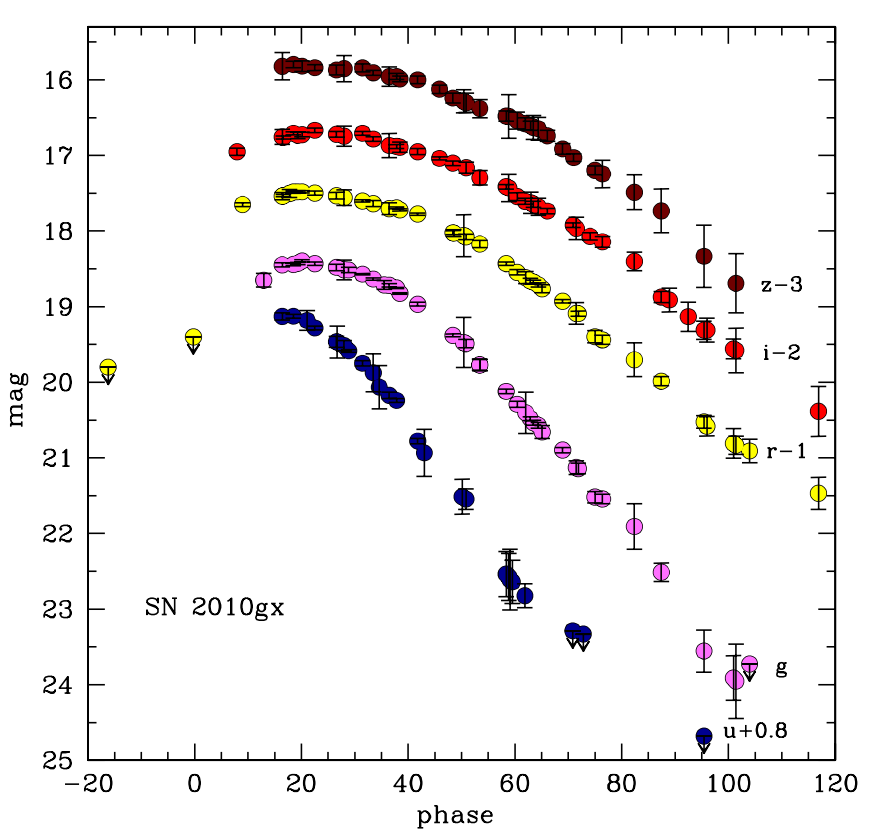

Figure 1. Observed ugriz light curves of SN 2010gx. The phase is from JD = $2,455,260$, used as an indicative explosion epoch. Detection limits from Mahabal et al. (2010) and Quimby et al. (2010a) are included.

(A color version of this figure is available in the online journal.)

The observed light curves, calibrated using 10 SDSS stars in the field of the transient, are shown in Figure 1. The transient was discovered in the rising phase, and its light curves are asymmetric. The pre-discovery limit of March 4 (Quimby et al. 2010a) indicates that SN 2010gx experienced a fast rise to maximum, followed by a slower magnitude decline. A similar asymmetry was also observed in the light curve of SN 2005ap (Quimby et al. 2007). The photometric evolution of SN 2010gx is somewhat different from the bell-like shape observed in the slow-evolving light curve of SCP-06F6 (Barbary et al. 2009). Assuming negligible host galaxy reddening (Galactic reddening of $E(B-V)=0.04$ mag; Schlegel et al. 1998) and accounting for redshift effects, ${ }^{16}$ an absolute rest-frame peak magnitude of $M_{B} \approx-21.2$ (Vega system) is determined for SN 2010gx. In Figure 2, we compare the rest-frame, $B$-band absolute light curve of SN 2010gx with those of a few ultra-bright events and classical type $\mathrm{Ib} / \mathrm{c} \mathrm{SNe}$, including broad-lined energetic SNe Ic. The epoch of the $B$-band maximum for SN 2010gx was computed with a low-order polynomial fit to the light curve and found to be at JD $=2455283 \pm 2$. The absolute peak magnitude of SN 2010gx is slightly fainter than that of SN 2005ap, ${ }^{17}$ while no direct comparison is possible with the peculiar SN 2007bi (observed well past-maximum in the $B$ band; Young et al. 2010) and SCP-06F6 (Barbary et al. 2009, for which a reliable restframe absolute light curve was computed only for the $u$ band). However, SN 2010gx appears to be 2.5-5 mag brighter than $\mathrm{SNe} \mathrm{Ib} / \mathrm{c}$ reported in Figure 2, and its overall evolution is much slower than that of normal $\mathrm{Ib} / \mathrm{c}$ events, although faster than that experienced by SN 2007bi.

A major difference between the light curves of $\mathrm{SNe} \mathrm{Ib} / \mathrm{c}$ and $\mathrm{SN} 2010 \mathrm{gx}$ is the apparent lack of a radioactive tail, in analogy to that observed in the case of other objects of the

\footnotetext{
16 Time dilation and $K$-correction (computed using our SN 2010gx spectra), with the latter producing Johnson $B$ band from observed SDSS $r$ band.

17 Note that only unfiltered photometry is available for SN 2005ap, calibrated using USNO-B1.0 R2 magnitudes (Quimby et al. 2007).
} 
Table 1

Observed (Non-K-corrected) Photometry of SN 2010gx (AB mag) Plus Associated Errors

\begin{tabular}{|c|c|c|c|c|c|c|c|c|}
\hline Date & JD & Phase $^{\mathrm{a}}$ & $u$ & $g$ & $r$ & $i$ & $z$ & Telescope \\
\hline 2010 Mar 12 & 2455267.95 & -12.2 & & & & $18.95(0.05)$ & & PS1 \\
\hline 2010 Mar 13 & 2455268.99 & -11.4 & & & $18.65(0.03)$ & & & PS1 \\
\hline 2010 Mar 17 & 2455272.96 & -8.2 & & $18.65(0.09)$ & & & & PS1 \\
\hline 2010 Mar 20 & 2455276.42 & -5.3 & $18.33(0.05)$ & $18.45(0.03)$ & $18.54(0.05)$ & $18.76(0.10)$ & $18.82(0.18)$ & LT \\
\hline 2010 Mar 21 & 2455276.61 & -5.2 & & & $18.53(0.02)$ & $18.76(0.02)$ & & NOT \\
\hline 2010 Mar 22 & 2455277.77 & -4.3 & & & $18.50(0.05)$ & & & GS \\
\hline 2010 Mar 22-23 & 2455278.50 & -3.7 & $18.33(0.02)$ & $18.44(0.02)$ & $18.48(0.02)$ & $18.71(0.02)$ & $18.80(0.04)$ & LT \\
\hline 2010 Mar 23 & 2455279.31 & -3.0 & & $18.43(0.02)$ & $18.48(0.02)$ & $18.73(0.05)$ & & LOT \\
\hline 2010 Mar 24 & 2455280.14 & -2.3 & & $18.40(0.02)$ & $18.48(0.02)$ & $18.72(0.03)$ & $18.82(0.07)$ & LOT \\
\hline 2010 Mar 25 & 2455281.07 & -1.6 & $18.38(0.13)$ & & & & & UVOT \\
\hline 2010 Mar 27 & 2455282.52 & -0.4 & $18.48(0.03)$ & $18.43(0.02)$ & $18.50(0.03)$ & $18.67(0.03)$ & $18.84(0.04)$ & $\mathrm{LT}$ \\
\hline 2010 Mar 31 & 2455286.58 & 2.9 & $18.66(0.07)$ & $18.48(0.04)$ & $18.53(0.05)$ & $18.72(0.04)$ & $18.87(0.07)$ & LT \\
\hline 2010 Mar 31 & 2455286.70 & 3.0 & $18.67(0.21)$ & & & & & UVOT \\
\hline 2010 Apr 1 & 2455287.99 & 4.1 & $18.72(0.07)$ & $18.51(0.13)$ & $18.56(0.10)$ & $18.75(0.13)$ & $18.85(0.17)$ & FTN \\
\hline 2010 Apr 2 & 2455288.85 & 4.8 & $18.78(0.02)$ & $18.52(0.04)$ & & & & FTN \\
\hline 2010 Apr 4 & 2455291.45 & 6.9 & $18.95(0.04)$ & $18.57(0.01)$ & $18.60(0.02)$ & $18.71(0.02)$ & $18.84(0.05)$ & $\mathrm{LT}$ \\
\hline 2010 Apr 6 & 2455293.48 & 8.5 & $19.08(0.25)$ & $18.64(0.02)$ & $18.64(0.04)$ & $18.78(0.03)$ & $18.91(0.03)$ & LT \\
\hline 2010 Apr 8 & 2455294.59 & 9.4 & $19.26(0.29)$ & & & & & UVOT \\
\hline 2010 Apr 9 & 2455295.53 & 10.2 & & $18.71(0.06)$ & & & & GS \\
\hline 2010 Apr 9 & 2455296.46 & 10.9 & $19.37(0.04)$ & $18.72(0.02)$ & $18.70(0.08)$ & $18.87(0.16)$ & $18.96(0.13)$ & LT \\
\hline 2010 Apr 11 & 2455297.84 & 12.1 & $19.44(0.02)$ & $18.75(0.01)$ & $18.69(0.01)$ & $18.88(0.03)$ & $18.96(0.04)$ & FTN \\
\hline 2010 Apr 11 & 2455298.48 & 12.6 & & $18.82(0.01)$ & $18.72(0.01)$ & $18.89(0.07)$ & $18.99(0.04)$ & $\mathrm{LT}$ \\
\hline 2010 Apr 15 & 2455301.80 & 15.3 & $19.98(0.04)$ & $18.97(0.03)$ & $18.78(0.02)$ & $18.95(0.04)$ & $19.00(0.05)$ & FTN \\
\hline 2010 Apr 16 & 2455303.04 & 16.3 & $20.13(0.31)$ & & & & & UVOT \\
\hline 2010 Apr 19 & 2455305.88 & 18.6 & & & & $19.04(0.02)$ & $19.12(0.06)$ & FTN \\
\hline 2010 Apr 21 & 2455308.38 & 20.6 & & $19.38(0.02)$ & $19.02(0.03)$ & $19.10(0.03)$ & $19.24(0.07)$ & $\mathrm{LT}$ \\
\hline 2010 Apr 22 & 2455308.58 & 20.8 & & & $19.03(0.05)$ & & & GS \\
\hline 2010 Apr 23 & 2455310.13 & 22.1 & $20.71(0.23)$ & & & & & UVOT \\
\hline 2010 Apr 23 & 2455310.40 & 22.3 & & $19.48(0.33)$ & $19.06(0.28)$ & & $19.28(0.15)$ & LT \\
\hline 2010 Apr 24 & 2455310.84 & 22.6 & $20.75(0.14)$ & $19.49(0.05)$ & $19.08(0.04)$ & & & FTN \\
\hline 2010 Apr 24 & 2455310.88 & 22.7 & & & & $19.16(0.08)$ & $19.30(0.13)$ & FTN \\
\hline 2010 Apr 26 & 2455313.42 & 24.7 & & $19.77(0.07)$ & & & & $\mathrm{LT}$ \\
\hline 2010 Apr 26 & 2455313.44 & 24.7 & & $19.77(0.08)$ & $19.17(0.05)$ & $19.30(0.10)$ & $19.38(0.12)$ & LT \\
\hline 2010 May 1 & 2455318.39 & 28.8 & $21.74(0.30)$ & $20.12(0.03)$ & $19.43(0.02)$ & $19.41(0.03)$ & $19.48(0.07)$ & $\mathrm{LT}$ \\
\hline 2010 May 2 & 2455318.84 & 29.1 & $21.77(0.31)$ & & & $19.43(0.18)$ & $19.49(0.29)$ & FTN \\
\hline 2010 May 2 & 2455319.09 & 29.3 & $21.81(0.40)$ & & & & & UVOT \\
\hline 2010 May 3 & 2455319.54 & 29.7 & $21.84(0.29)$ & & & & & LT \\
\hline 2010 May 3 & 2455320.43 & 30.4 & & $20.29(0.04)$ & $19.55(0.04)$ & $19.54(0.05)$ & $19.54(0.11)$ & LT \\
\hline 2010 May 5 & 2455321.88 & 31.6 & $22.02(0.16)$ & & & & & FTN \\
\hline 2010 May 5 & 2455321.94 & 31.7 & & & & $19.61(0.04)$ & $19.58(0.08)$ & FTS \\
\hline 2010 May 5 & 2455322.03 & 31.7 & & $20.40(0.27)$ & $19.62(0.08)$ & & & FTS \\
\hline 2010 May 6 & 2455322.88 & 32.4 & & $20.48(0.03)$ & $19.66(0.04)$ & & & FTS \\
\hline 2010 May 6 & 2455322.94 & 32.5 & & & & & $19.61(0.06)$ & FTS \\
\hline 2010 May 6 & 2455323.02 & 32.5 & & & & $19.63(0.14)$ & & FTS \\
\hline 2010 May 6 & 2455323.48 & 32.9 & & $20.54(0.03)$ & $19.68(0.03)$ & $19.65(0.04)$ & $19.63(0.16)$ & $\mathrm{LT}$ \\
\hline 2010 May 7 & 2455324.38 & 33.6 & & $20.57(0.04)$ & $19.71(0.03)$ & $19.68(0.11)$ & $19.65(0.15)$ & LT \\
\hline 2010 May 8 & 2455325.11 & 34.2 & & $20.66(0.08)$ & $19.76(0.05)$ & & & FTS \\
\hline 2010 May 9 & 2455326.09 & 35.0 & & & & $19.74(0.03)$ & $19.74(0.07)$ & FTS \\
\hline 2010 May 12 & 2455328.95 & 37.4 & & $20.90(0.04)$ & $19.93(0.02)$ & & $19.91(0.07)$ & FTS \\
\hline 2010 May 14 & 2455330.84 & 38.9 & $>22.49$ & & & & & FTN \\
\hline 2010 May 14 & 2455330.90 & 38.9 & & & & $19.92(0.03)$ & & FTS \\
\hline 2010 May 14 & 2455331.01 & 39.0 & & & & & $20.03(0.05)$ & FTS \\
\hline 2010 May $14-15$ & 2455331.50 & 39.4 & & $21.13(0.09)$ & $20.09(0.14)$ & $19.97(0.15)$ & & $\mathrm{LT}$ \\
\hline 2010 May 15 & 2455331.88 & 39.7 & & $21.14(0.07)$ & $20.09(0.03)$ & & & FTS \\
\hline 2010 May 16 & 2455332.82 & 40.5 & $>22.53$ & & & & & FTN \\
\hline 2010 May 17 & 2455334.09 & 41.5 & & & & $20.07(0.05)$ & & FTS \\
\hline 2010 May 18 & 2455335.02 & 42.3 & & $21.52(0.08)$ & $20.40(0.08)$ & & $20.20(0.06)$ & FTS \\
\hline 2010 May 19 & 2455336.40 & 43.4 & & $21.54(0.06)$ & $20.44(0.06)$ & $20.14(0.07)$ & $20.25(0.18)$ & $\mathrm{LT}$ \\
\hline 2010 May 25 & 2455342.39 & 48.3 & & $21.91(0.30)$ & $20.70(0.22)$ & $20.40(0.12)$ & $20.49(0.23)$ & $\mathrm{LT}$ \\
\hline 2010 May 30 & 2455347.42 & 52.4 & & $22.51(0.12)$ & $20.99(0.06)$ & $20.87(0.07)$ & $20.73(0.29)$ & LT \\
\hline 2010 Jun 1 & 2455348.95 & 53.6 & & & & $20.91(0.16)$ & & FTS \\
\hline 2010 Jun 4 & 2455352.47 & 56.5 & & & & $21.13(0.19)$ & & GS \\
\hline 2010 Jun 8 & 2455355.43 & 58.9 & $>23.88$ & $23.55(0.28)$ & $21.52(0.09)$ & $21.31(0.12)$ & $21.33(0.41)$ & WHT \\
\hline 2010 Jun 8 & 2455355.98 & 59.3 & & & $21.58(0.13)$ & $21.31(0.16)$ & & FTS \\
\hline 2010 Jun 13 & 2455360.97 & 63.4 & & $23.91(0.30)$ & $21.81(0.20)$ & $21.56(0.13)$ & & FTS \\
\hline
\end{tabular}


Table 1

(Continued)

\begin{tabular}{|c|c|c|c|c|c|c|c|c|}
\hline Date & JD & Phase $^{\mathrm{a}}$ & $u$ & $g$ & $r$ & $i$ & $z$ & Telescope \\
\hline 2010 Jun 13 & 2455361.42 & 63.8 & & $23.95(0.49)$ & $21.83(0.12)$ & $21.58(0.29)$ & $21.69(0.39)$ & LT \\
\hline 2010 Jun 16 & 2455363.98 & 65.8 & & $>23.73$ & $21.91(0.16)$ & & & FTS \\
\hline 2010 Jun 29 & 2455376.88 & 76.3 & & & $22.47(0.21)$ & $22.38(0.33)$ & & FTS \\
\hline
\end{tabular}

Notes. UVOT- $u$ Swift, and R and I NOT data have been converted to SDSS magnitudes. Column 2 reports the phases with respect to the $B$-band maximum. PS1 $=1.8 \mathrm{~m}$ Pan-STARRS1; GS $=8.1 \mathrm{~m}$ Gemini South + GMOS; LT $=2.0 \mathrm{~m}$ Liverpool Telescope +RatCam; NOT $=2.56 \mathrm{~m}$ Nordic Optical Telescope + ALFOSC; LOT $=1.0 \mathrm{~m}$ Lulin Telescope; UVOT $=$ Swift + UVOT; FTN $=2.0 \mathrm{~m}$ Faulkes Telescope North + MEROPE; FTS $=2.0 \mathrm{~m}$ Faulkes Telescope South +MEROPE; WHT $=4.2 \mathrm{~m}$ William Herschel Telescope +ACAM.

${ }^{\text {a }}$ Corrected for time dilation.

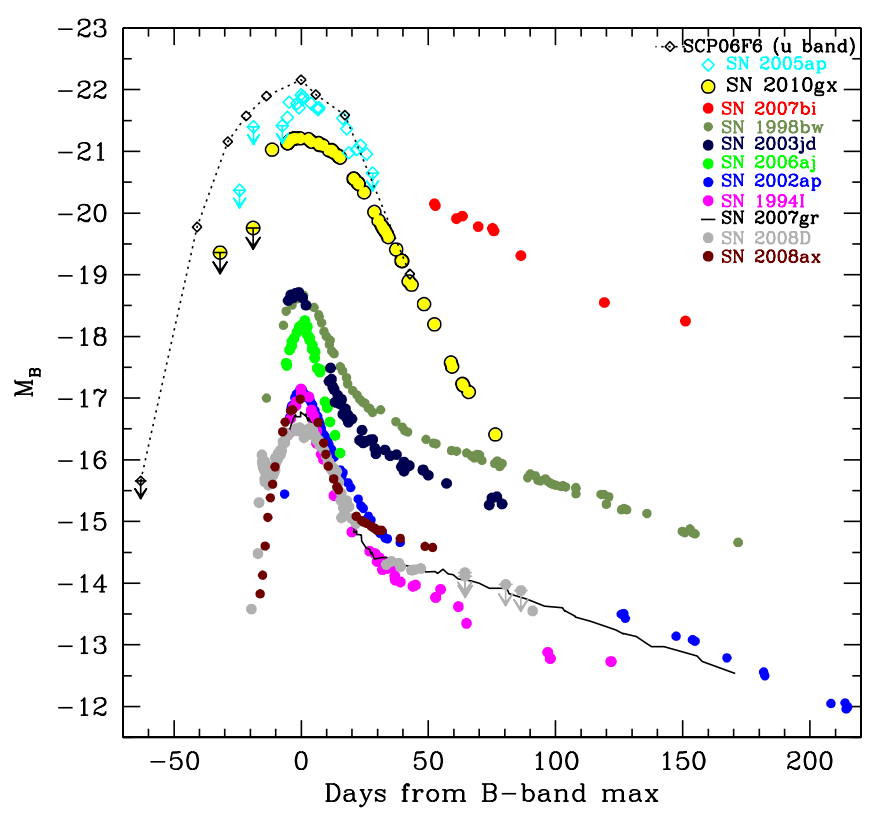

Figure 2. $B$-band absolute light curves of SN 2010gx (Vega system) and a number of ultra-bright events and canonical stripped-envelope SNe, including the type Ic SNe 1994I (Richmond et al. 1996, and references therein), 2002ap (Pandey et al. 2002; Foley et al. 2003; Yoshii et al. 2003; Tomita et al. 2006), 2006aj (Campana et al. 2006; Cobb et al. 2006; Mirabal et al. 2006; Pian et al. 2006; Sollerman et al. 2006), 2003jd (Valenti et al. 2008a), and 1998bw (Galama et al. 1998; McKenzie \& Schaefer 1999; Sollerman et al. 2000; Patat et al. 2001); the type Ib SNe 2007gr (Valenti et al. 2008b; Hunter et al. 2009) and 2008D (Mazzali et al. 2008; Soderberg et al. 2008; Modjaz et al. 2009); the type IIb SN 2008ax (Pastorello et al. 2008). B-band light curves for the luminous SNe 2005ap, 2010gx, and 2007bi are obtained correcting the observed broadband photometry for time dilation and differences in effective rest-frame band ( $K$-correction). The high redshift of SCP-06F6 $(z=1.189)$ did not allow us to compute a realistic $B$-band absolute light curve, so we estimated the $u$-band light curve (Vega system) from the $i_{775}$-band photometry of Barbary et al. (2009). $K$-corrections for the luminous objects were computed using the spectra published by Barbary et al. (2009), Quimby et al. (2007), Young et al. (2010), and this Letter.

(A color version of this figure is available in the online journal.)

Quimby et al. sample. However, with the data collected so far, we cannot exclude the possibility that the light curve flattens onto a radioactive tail at later epochs. In that case, the expected amount of ${ }^{56} \mathrm{Ni}$ ejected by SN $2010 \mathrm{gx}$ would be comparable (or only marginally higher, e.g., $\lesssim 1 M_{\odot}$ ) to that of type $\mathrm{Ib} / \mathrm{c} \mathrm{SNe}$.

\subsection{Spectroscopy}

A sequence of spectra of SN 2010gx was obtained with the $2.56 \mathrm{~m}$ Nordic Optical Telescope and the $4.2 \mathrm{~m}$ William Herschel Telescope (La Palma, Canary Islands), and the $8.1 \mathrm{~m}$ Gemini South Telescope (Cerro Pachón, Chile). Pre-maximum

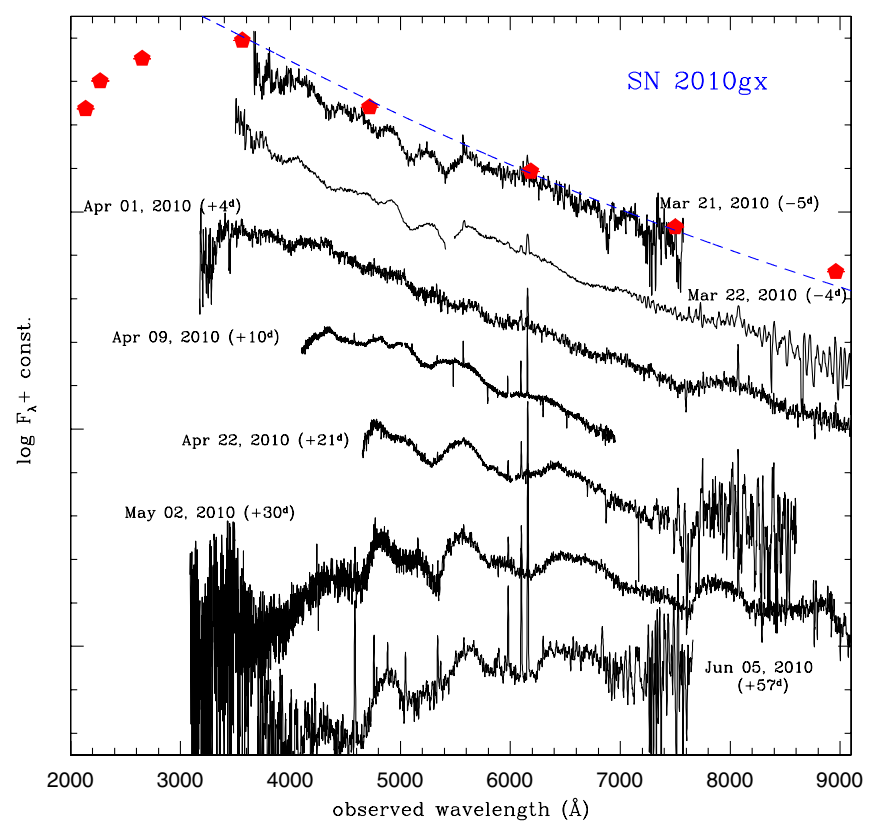

Figure 3. Spectral evolution of SN 2010gx. All spectra are in the observed frame. The phases in parentheses are relative to the $B$-band maximum. The red pentagons represent the observed spectral energy distribution calculated using Swift-UVOT (PI: Quimby) and Liverpool Telescope photometry obtained between March 19 and March 20. Early UVOT magnitudes (Vega system) are: $u w 2=18.69 \pm 0.07(\mathrm{JD}=2,455,276.18), u m 2=18.21 \pm 0.08(\mathrm{JD}=$ $2,455,274.64)$, and $u w 1=17.71 \pm 0.06(\mathrm{JD}=2,455,274.65)$. The deviation of the ultra-violet contribution from a hot blackbody continuum $\left(T_{\mathrm{bb}}=15,000 \mathrm{~K}\right.$, dotted blue line) is probably due to line blanketing in that region.

(A color version of this figure is available in the online journal.)

spectra obtained on March 21 and 22 show a very blue continuum (with a blackbody temperature $T_{\mathrm{bb}}=15,000 \pm$ $1700 \mathrm{~K}$ ) with broad absorption features below $\sim 5700 \AA$ (Figure 3). Weak, narrow emission lines ( $\mathrm{H} \alpha, \mathrm{H} \beta$, and the [O III] doublet at $4959,5007 \AA$ ) of the host galaxy are also visible, confirming the identification of the broad features as $\mathrm{O}$ II, also identified by Quimby et al. (2010b) in the spectra of SN 2005ap (Figure 4, top).

A spectrum obtained on April 1 ( +4 days) is still blue $\left(T_{\mathrm{bb}} \simeq 13,000 \pm 1200 \mathrm{~K}\right)$ but is almost featureless. A significant evolution of the spectra of SN 2010gx then occurred at 1020 days after peak. At these epochs the spectra show very broad P-Cygni absorptions of Ca II, Fe II, and Si II, very similar to those observed in spectra of young SNe Ic (Filippenko 1997). The subsequent spectrum, obtained on May 2 (+30 days), is markedly more similar to those of SNe Ic soon after maximum light. Finally, a further spectrum was obtained on June 5 (+57 days) and showed only a mild evolution in the spectral features. 


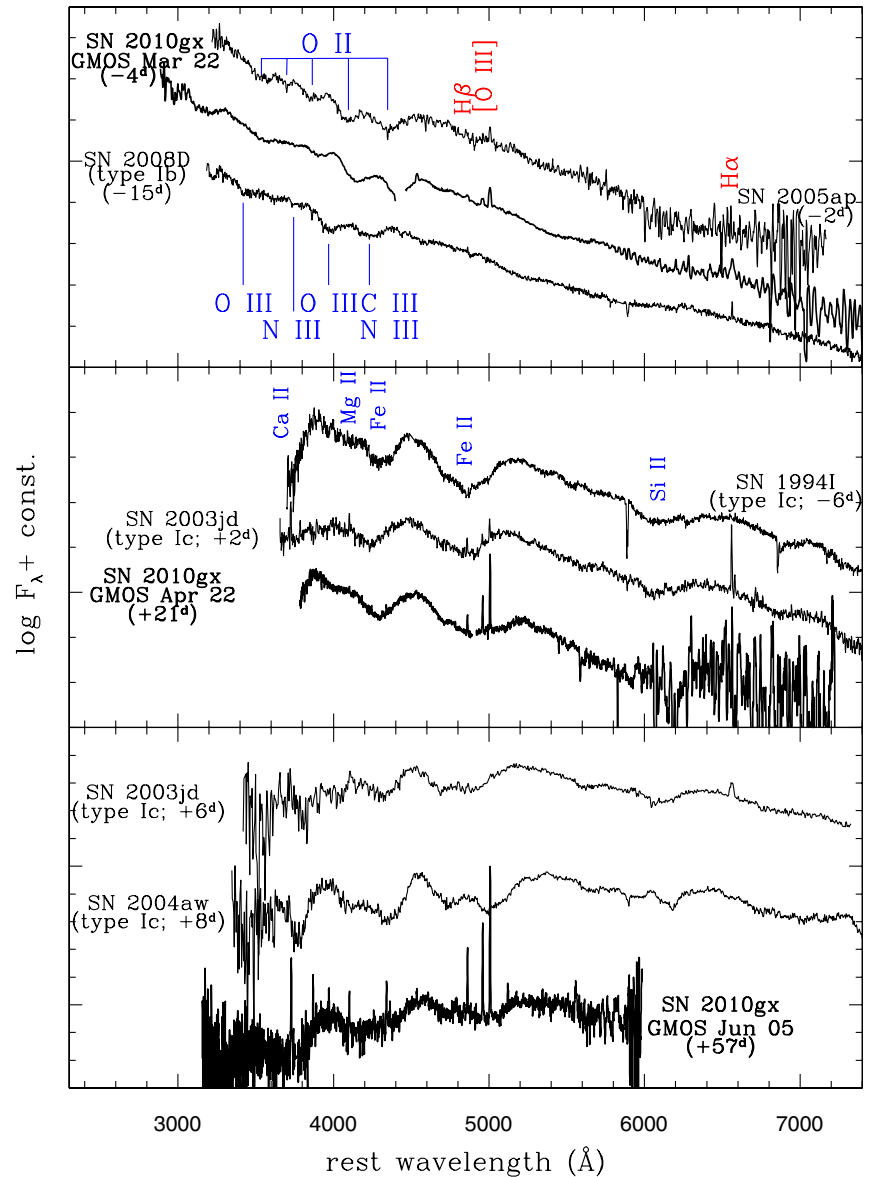

Figure 4. Top: comparison of early-time spectra of SNe 2010gx and 2005ap (Quimby et al. 2007) with one of the type Ib SN 2008D associated with the X-ray transient (XRT) 080109 (obtained +1.84 days from XRT 080109; Modjaz et al. 2009). All spectra show similar absorption bumps between $3500 \AA$ and $4500 \AA$, although slightly shifted in the three spectra. These have been tentatively identified as O II features (Quimby et al. 2010b) and blends of O III/N III/C III (Modjaz et al. 2009). Middle: comparison of the April 22 spectrum of SN 2010gx with spectra of the Ic SN 1994I (Baron et al. 1996) and the moderately broadlined SN 2003jd (Valenti et al. 2008a) around maximum. Now the spectrum of SN 2010gx is dominated by broad absorptions at about $3700 \AA$ (Ca II H and $\mathrm{K}), 4300 \AA$ (Mg II, blended with Fe II), $4900 \AA$ (Fe II, plus possibly Mg I), and $6100 \AA$ (Si II). Bottom: comparison of the June 5 spectrum of SN 2010gx with later spectra of the type Ic SNe 2003jd (Valenti et al. 2008a) and 2004aw (Taubenberger et al. 2006). The phases labeled in figure are from the $B$-band maximum

(A color version of this figure is available in the online journal.)

The spectral evolution of SN 2010gx from an SCP-06F6like event to a type Ic SN provides an unexpected clue for understanding the evolutionary path of this class of transients. In order to produce $\mathrm{O}$ II features (together with the $\mathrm{Si}$ III, $\mathrm{C}$ II, and Mg II lines observed in spectra of other objects of this family, see Quimby et al. 2010a, and Figure 4, top), high photospheric temperatures are necessary. Interestingly, Modjaz et al. (2009) noted a short-life "W" feature in a very early spectrum of the type Ib SN 2008D. That feature, visible in a spectrum taken 1.84 days after the X-ray flash 080109 associated with the SN, disappeared 1 day later. Modjaz et al. (2009) noted striking similarity with the early-time spectrum of SN 2005ap and (following Quimby et al. 2007) tentatively identified such short-life features as a combination of O III, N III, and C III lines. However, the "W" feature in SN 2008D is slightly blueshifted compared to the analogous feature visible in the early spectra of SNe 2005ap and 2010gx. Therefore, fleeting lines due to ionized intermediatemass elements could be common in very early spectra of some type $\mathrm{Ib} / \mathrm{c} \mathrm{SNe}$. However, these lines are visible for several weeks after the explosion in SCP-06F6-like objects, which is likely due to higher densities and temperatures of the ejecta which persist for longer than in canonical $\mathrm{SNe} \mathrm{Ib/c.}$

As the SN expands, the ejecta become cooler and other broad lines appear (Ca II, Mg II, Fe II, and Si II). These features are commonly visible in $\mathrm{Ib} / \mathrm{c}$ spectra around maximum (Filippenko 1997). In Figure 4 (middle), a later spectrum of SN 2010gx (+21 days) is compared with a pre-maximum spectrum of the normal type Ic SN 1994I (Baron et al. 1996) and a slightly postmaximum spectrum of the broad-line Ic SN 2003jd (Valenti et al. 2008a). The striking similarity among these three spectra is a confirmation that SN 2010gx (and possibly all SCP06F6-like objects) should be considered spectroscopically as $\mathrm{SNe}$ Ic, although with rather extreme photometric properties (Section 3.1). The similarity with normal stripped-envelope $\mathrm{SNe}$ is even more evident in the comparison of the last spectrum of SN 2010gx (June 5) with spectra of the type Ic SNe 2003jd (Valenti et al. 2008a) and 2004aw (Taubenberger et al. 2006) obtained about 1 week after their $B$-band peaks.

\section{THE NATURE OF ULTRA-BRIGHT EVENTS}

SN 2010gx provides important clues to understand the nature of ultra-bright events. Its spectro-photometric similarities with this family is well established: high luminosity, slow-evolving light curves, similar spectral properties, and faint host galaxies. The spectral evolution of SN 2010gx now links this family of transients to the more common type $\mathrm{Ib} / \mathrm{c} \mathrm{SNe}$, and by implication the progenitor stars. The overall spectral evolution is indeed similar to that of $\mathrm{SNe} \mathrm{Ib} / \mathrm{c}$, although $\mathrm{SN} 2010 \mathrm{gx}$ spectroscopically evolved on a much longer timescale. The observed parameters of SN 2010gx present several problems in interpreting the explosion. Its impressive luminosity at maximum and slower evolution could simply be interpreted as implying large photospheric radii $\left(L \sim R^{2} T^{4}\right)$ and large ejecta masses $\left(\tau \sim(\kappa M / v)^{1 / 2}\right.$; for radiative diffusion from a sphere). The energy source for $\mathrm{SNe} \mathrm{Ib} / \mathrm{c}$ is the decay of radioactive isotopes, but the 80 day long post-peak decline of SN 2010gx (Figure 2) is too steep to be due to ${ }^{56} \mathrm{Co}$ decay. It is plausible that a radioactive tail could be detected at later phases, if the light curve flattens to the luminosity of the type Ic SN 1998bw. But this would imply $\lesssim 1 M_{\odot}$ of ${ }^{56} \mathrm{Co}$ decaying into ${ }^{56} \mathrm{Fe}$ to power the tail luminosity, and such a moderate mass of ${ }^{56} \mathrm{Ni}$ cannot account for the high bolometric luminosity at peak, which is $\sim(3-4) \times 10^{44} \mathrm{erg} \mathrm{s}^{-1}$. Assuming that the bolometric luminosity from the earliest PTF detection to our earliest multi-band observation was constant, the energy radiated by SN 2010gx during the first 100 days is $\sim 6 \times 10^{51} \mathrm{erg}$.

The peak luminosity of SN $2010 \mathrm{gx}_{\mathrm{x}}$ is quite similar to that of SN 2007bi, but the light curve and spectral evolution are completely different (Gal-Yam et al. 2009; Young et al. 2010). The slow decay time and appearance of strong [Fe II] lines in SN 2007bi suggested a kinetic energy of few $\times 10^{53} \mathrm{erg}$, very massive ejecta, and 3-6 $M_{\odot}$ of ${ }^{56} \mathrm{Ni}$ synthesized. Gal-Yam et al. (2009) postulated that this was the explosion of a $100 M_{\odot}$ core in a pair-instability SN. While this possibility was also noted by Young et al. (2010), the gravitational collapse of the $\mathrm{C}+\mathrm{O}$ core of a massive star $\left(M_{\mathrm{ZAMS}}=50-100 M_{\odot}\right)$ is a viable mechanism (as recently calculated by Moriya et al. 2010). Whatever the explosion scenario, a large amount of ${ }^{56} \mathrm{Ni}$ is necessary. However, SN $2010 \mathrm{gx}$ is markedly different 
in its properties, particularly the more rapid decay in its light curve indicates that the pair-instability scenario and a large ${ }^{56} \mathrm{Ni}$ production are unlikely to be the explanation.

The apparent lack of any evidence of light curve flattening to a radioactive tail led Quimby et al. (2010b) to favor the pulsational pair-instability eruption scenario over a genuine $\mathrm{SN}$ explosion for SN 2005ap, SCP-06F6, and other PTF SNe. In the pulsational pair-instability model, the luminosity is generated by the collision of shells of material ejected at different times by the pulsations. Little or no ${ }^{56} \mathrm{Ni}$ powers the light curve for long periods. The outbursts are expected to be energetic, reaching very high peak luminosities and creating hot $\left(T_{\text {eff }} \approx 25,000 \mathrm{~K}\right)$, optically thick photospheres (Woosley et al. 2007). All of this is consistent with the parameters observed in ultra-bright $\mathrm{SNe}$, and our well sampled light curve of SN $2010 \mathrm{gx}$ is not too different from those calculated by Woosley et al. (2007). However, these models are for supergiant progenitors with large, extended $\mathrm{H}$-rich envelopes. The energy released in the pulsations is predicted to be $(0.005-2) \times 10^{51} \mathrm{erg}$, in most cases below $10^{51}$ erg. This is enough to eject the loosely bound envelope of an extended supergiant, but weather or not this mechanism could eject a substantial part of a more compact WR star (Dessart et al. 2010) remains to be calculated in detail. Additionally, we do not see any sign of interaction between dense gas shells in the form of narrow circumstellar lines. While the pulsational pair-instability model is appealing as it can produce the high luminosity, it needs further consideration to determine if it is physically viable for $\mathrm{H}$-free progenitor stars.

Another possibility, also discussed in Quimby et al. (2010b), is that ultra-bright $\mathrm{SNe}$ are powered by the spin down of newly born magnetars (Kasen \& Bildsten 2010; Woosley 2010). A magnetar with a moderate magnetic field $\left(B \approx 10^{14} \mathrm{G}\right)$ and spinning periods of $2-20 \mathrm{~ms}$ can produce peak luminosities similar to that observed in SN 2010gx $\left(\sim(3-4) \times 10^{44} \mathrm{erg} \mathrm{s}^{-1}\right)$. In addition, magnetar-powered SN models do not need large ${ }^{56} \mathrm{Ni}$ and total ejected masses (Kasen \& Bildsten 2010; Woosley 2010) and can show Ic SN features (Woosley 2010). However our extensive light curve coverage of SN 2010gx shows a faster decline than the model light curves, and our estimated photospheric temperatures are a factor 2-4 higher than model predictions.

The observational evidence presented in this Letter links SN 2010gx (and probably the entire family of transients described by Quimby et al. 2010b) with SNe Ic. The very luminous and broad light curve implies much larger ejecta masses than inferred even for the broad-lined SNe Ic $\left(\sim 8-15 M_{\odot}\right.$; Valenti et al. 2008a). The close similarity in the spectra implies that the progenitor was a massive WR star, but the energy source powering the remarkable luminosity is uncertain. In fact, we have an SN-like transient which does not comfortably match any of the known SN scenarios, i.e., core-collapse and ${ }^{56} \mathrm{Ni}$ powered explosion, pair-instability, pulsational pair-instability nor magnetar-powered event.

The key diagnostics in the future will be late-time photometric monitoring after solar conjunction and very early detection of new events. The presence of a late-time light curve tail with a slope roughly consistent with the ${ }^{56}$ Co decay could support a real SN explosion, and the early rise time can help to determine the progenitor radius and possibly signs of interaction between colliding shells. The recent suggestion that the most massive stars in the LMC may be up to $320 M_{\odot}$ (Crowther et al. 2010) could lead to more diverse SN progenitor populations than is currently appreciated (Smartt 2009).
The PS1 Surveys have been made possible through contributions of the Institute for Astronomy at the University of Hawaii in Manoa, the Pan-STARRS Project Office, the Max-Planck Society and its participating institutes, the Max Planck Institute for Astronomy, Heidelberg and the Max Planck Institute for Extraterrestrial Physics, Garching, The Johns Hopkins University, the University of Durham, the University of Edinburgh, the Queens University Belfast, the Harvard- Smithsonian Center for Astrophysics, and the Los Cumbres Observatory Global Telescope Network, Incorporated. This work was conducted as part of a EURYI scheme award.

\section{REFERENCES}

Barbary, K., et al. 2009, ApJ, 690, 1358

Baron, E., Hauschildt, P. H., Branch, D., Kirshner, R. P., \& Filippenko, A. V. 1996, MNRAS, 279, 799

Campana, S., et al. 2006, Nature, 442, 1008

Cobb, B. E., Bailyn, C. D., van Dokkum, P. G., \& Natarajan, P. 2006, ApJ, 645, L113

Crowther, P. A., Schnurr, O., Hirschi, R., Yusof, N., Parker, R. J., Goodwin, S. P., \& Abu Kassim, H. 2010, MNRAS, 408, 731

Dessart, L., Livne, E., \& Waldman, R. 2010, MNRAS, 405, 2113

Drake, A. J., et al. 2009, ApJ, 696, 870

Filippenko, A. V. 1997, ARA\&A, 35, 309

Foley, R., et al. 2003, PASP, 115, 1220

Galama, T. J., et al. 1998, Nature, 395, 670

Gal-Yam, A., et al. 2009, Nature, 462, 624

Gänsicke, B. T., Levan, A. J., Marsh, T. R., \& Wheatley, P. J. 2009, ApJ, 697, L129

Gezari, S., et al. 2009, ApJ, 690, 1313

Hunter, D. J., et al. 2009, A\&A, 508, 371

Kasen, D., \& Bildsten, L. 2010, ApJ, 717, 245

Law, N. M., et al. 2009, PASP, 121, 1395

Mahabal, A. A., \& Drake, A. J. 2010, ATel, 2508, 1

Mahabal, A. A., et al. 2010, ATel, 2490, 1

Mazzali, P. A., et al. 2008, Science, 321, 1185

McKenzie, E. H., \& Schaefer, B. E. 1999, PASP, 111, 964

Miller, A. A., et al. 2009, ApJ, 690, 1303

Mirabal, N., Halpern, J. P., An, D., Thorstensen, J. R., \& Terndrup, D. M. 2006, ApJ, 643, L99

Modjaz, M., et al. 2009, ApJ, 702, 226

Moriya, T., Tominaga, N., Tanaka, M., Maeda, K., \& Nomoto, K. 2010, ApJ, 717, L83

Pandey, S. B., Anupama, G. C., Sagar, R., Bhattacharya, D., Sahu, D. K., \& Pandey, J. C. 2002, MNRAS, 340, 375

Pastorello, A., et al. 2008, MNRAS, 389, 955

Pastorello, A., et al. 2010, ATel, 2504, 1

Patat, F., et al. 2001, ApJ, 555, 900

Pian, E., et al. 2006, Nature, 442, 1011

Quimby, R. M., Aldering, G., Wheeler, J. C., Höflich, P., Akerlof, C. W., \& Rykoff, E. S. 2007, ApJ, 668, L99

Quimby, R. M., Kulkarni, S. R., Ofek, E., Kasliwal, M. M., Levitan, D., GalYam, A., \& Cenko, S. B. 2010a, ATel, 2492, 1

Quimby, R. M., et al. 2010b, Nature, submitted (arXiv:0910.0059)

Rau, A., et al. 2009, PASP, 121, 1334

Richmond, M. W., et al. 1996, AJ, 111, 327

Rosswog, S., Ramirez-Ruiz, E., \& Hix, W. R. 2009, ApJ, 695, 404

Schlegel, D. J., Finkbeiner, D. P., \& Davis, M. 1998, ApJ, 500, 525

Smartt, S. J. 2009, ARA\&A, 47, 63

Soderberg, A. M., et al. 2008, Nature, 453, 469

Soker, N., Frankowski, A., \& Kashi, A. 2009, New Astron., 15, 189

Sollerman, J., Kozma, C., Fransson, C., Leibundgut, B., Lundqvist, P., Ryde, F., \& Woudt, P. 2000, ApJ, 537, L127

Sollerman, J., et al. 2006, A\&A, 454, 503

Taubenberger, S., et al. 2006, MNRAS, 371, 1459

Tomita, H., et al. 2006, ApJ, 644, 400

Valenti, S., et al. 2008a, MNRAS, 383, 1485

Valenti, S., et al. 2008b, ApJ, 673, L155

Woosley, S. E. 2010, ApJ, 719, L204

Woosley, S. E., Blinnikov, S., \& Heger, A. 2007, Nature, 450, 390

Yoshii, Y., et al. 2003, ApJ, 592, 467

Young, D. R., et al. 2010, A\&A, 512, 70 